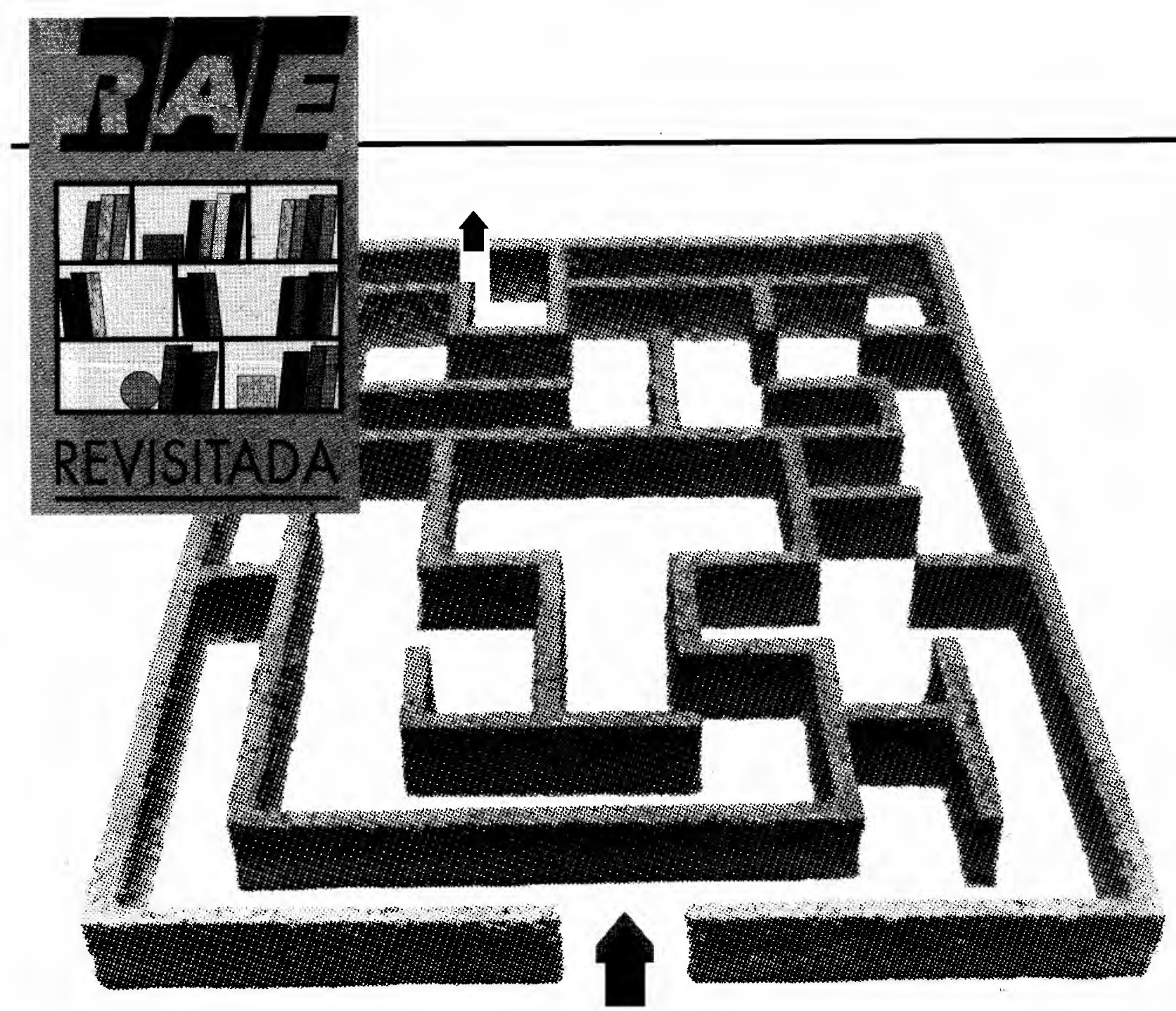

\title{
RESOLUÇÃO DE EQUAÇÕES BOOLEANAS: APLICAÇÃO A UM PROBLEMA DE DECISÃO EMPRESARIAL QUALITATIVA
}

Walter Del Picchia

Professor Titular do Departamento de Engenharia Eletrônica da Escola Politécnica da USP.

* RESUMO: Este trabalho é uma atualização do artigo publicado pelo autor na RAE em 1974 e tem por finalidade: a) definir o Problema da Decisão Qualitativa (PDQ); $b$ ) mostrar como certos tipos de problemas lógicos podem ser formulados como $P D Q s ; c)$ apresentar um método de resolução do $P D Q$, o qual utiliza resolução de equações booleanas; d) apresentar um processo original de resolução destas equações, o Algoritmo $R Q$, facilmente programável em computadores.

Os resultados apresentados são produto de trabalhos desenvolvidos no antigo Departamento de Engenharia de Eletricidade da EPUSP, os quais deram origem a diversas publicações, entre elas 1, 4, 5, 6, 7, 8, 9, 10, 11, 20, 23, 24, 25, 28, 29 (veja bibliografia no final do artigo).

Partes deste artigo são adaptações de trechos de livro do mesmo autor(11), onde se encontram os fundamentos teóricos, aplicações, sugestões para novos desenvolvimentos $e$ listagens para microcomputador do $S D B / 3$, um sistema de auxílio à tomada de decisões que resolve o $P D Q$.

* PALAVRAS-CHAVE: Problemas lógicos, equações booleanas, algoritmos numéricos, auxílio à decisão.

* ABSTRACT: The purpose of this paper is: a) to define the Qualitative Decision Problem $(Q D P) ; b)$ to show how a kind of logical problems can be formulated as QDPs; c) to introduce a method of resolution of the QDP, which utilizes resolution of boolean equations; d) to introduce the $R Q \mathrm{Al}$ gorithm, which is an original process of resolution of this equations.

* KEY WORDS: Logical problems, boolean equations, numerical algorithms, support to decision.

São Paulo, 33(4):54-65

Jul./Ago. 1993 


\section{PROBLEMA A RESOLVER}

Desejamos obter a solução do seguinte problema (21):

"A diretoria de uma firma manufatureira reuniu-se para decidir sobre a política de seus futuros negócios, em função de seus objetivos. Discutiram as relações entre o dinheiro gasto em propaganda, o preço das mercadorias manufaturadas, a percentagem das comissões dos vendedores e as possíveis mudanças para um tipo mais aperfeiçoado de seus produtos, e também os vários efeitos que suas decisões acarretariam quanto ao lucro total da firma e quanto à quantidade do produto vendido. Os objetivos da firma foram definidos: obter grande lucro e/ou vender grandes quantidades de artigos manufaturados, para levar a operação da fábrica à sua máxima capacidade possível. Todos os diretores concordaram nos cinco pontos seguintes:

I) Se a política da empresa for, ou aperfeiçoar o produto, ou cortar anúncios onerosos, ou pagar aos vendedores uma comissão mais elevada, ou elevar o preço das mercadorias vendidas a varejo, com a venda de um pequeno número de artigos, em qualquer desses casos, então o lụcro será pequeno.

II) Se grandes somas forem gastas em propaganda, sem alteração no tipo dos produtos, ou se houver mudança para um tipo mais aperfeiçoado e redução na dotação para a propaganda, então não haverá, simultaneamente, um grande volume de vendas e grandes lucros.

III) Se a firma tiver grande lucro ou se seu volume de vendas for elevado, então o preço dos artigos no varejo será baixo, e o gasto em propaganda, elevado; ou então o preço da venda a varejo será baixo, com uma pequena dotação para a propaganda, e haverá uma mudança para um tipo melhor de produto, com uma baixa comissão para os vendedores.

IV) Se o preço de venda a varejo for baixo, se não houver corte na despesa com propaganda e se houver comissões elevadas para os vendedores, ou se o preço de venda e as comissões forem baixos, com uma mudança para aperfeiçoar o produto, então uma grande quantidade de artigos será vendida.

V) Se houver um grande gasto em propaganda, com uma mudança para um produto aperfeiçoado, mas com o preço de venda conservado baixo e com muitos artigos vendidos, então haverá um lucro elevado.

Com essas regras gerais como guia, qual deverá ser a política de tomada de decisões da diretoria para se atingirem os seguintes objetivos:

a) obter grande margem de lucro;

b) obter grande volume de vendas;

c) obter simultaneamente grande lucro e grande volume de vendas?"

Está implícito que as respostas são desejadas em função de: aperfeiçoar o produto, gastar muito em propaganda, pagar altas comissões, elevar o preço de venda.

\section{CÁLCULO PROPOSICIONAL E ÁLGEBRA BOOLEANA}

Para equacionar o problema acima necessitamos introduzir alguns conceitos. $\mathrm{O}$ problema proposto pertence ao Cálculo Proposicional clássico e pode ser equacionado com auxílio da Álgebra Booleana Binária.

Nossa resolução se aplica a problemas da lógica que possam ser modelados pelo Cálculo Proposicional clássico (com extensão para o Cálculo de Predicados). Apesar de limitados $(14,15,16)$, o Cálculo Proposicional e o Cálculo de Predicados clássicos prestam-se para a análise de inúmeros problemas da vida real.

Embora apreciável esforço esteja sendo feito para o processamento de linguagens naturais, atualmente a formalização de problemas constituídos por frases nestas linguagens exige uma modelagem prévia das frases. As expressões do Cálculo Proposicional clássico, adiante estudadas, constituem um possível modelo, uma representação esquematizada destas frases. 
E a modelagem tem a finalidade de reduzir a complexidade delas, facilitando seu entendimento e manipulação.

Por exemplo, as ambigüidades e as exceções às regras presentes na linguagem natural, embora toleráveis (ou até desejáveis) num contexto literário, na formulação que propomos, devem ser eliminadas, antes de se traduzirem as idéias em expressões do Cálculo Proposicional clássico, para tornar os textos passíveis de tratamento formal. É necessário, assim, que seja selecionada (para ser traduzida em expressões do Cálculo Proposicional) uma única dentre as possíveis interpretações da frase original, e que corresponda à interpretação desejada pelo usuário.

Este processo de modelagem, porém, não é tranqüilo, surgindo inúmeras dificuldades, tais como: o mesmo texto em linguagem natural pode dar origem a diversos significados; as frases necessitam ser simplificadas, podendo levar a interpretações diferentes das originalmente desejadas; a riqueza da linguagem é esterilizada com a necessidade de tornar os enunciados precisos. Além disso, cuidados são essenciais para uma "tradução adequada" (que represente as intenções do usuário).

Desse modo, o problema se coloca na passagem das frases em linguagem natural para as expressões padronizadas do Cálculo Proposicional clássico, visto que as várias dificuldades acima citadas ocorrem nesta passagem. As frases são "traduzidas" num certo contexto, de acordo com uma seleção feita pelo usuário entre as possíveis traduções. Estas, às vezes, surpreendem-no, divergindo das intenções iniciais e levando a um processo de tentativa e erro, até que ele encontre a tradução precisa (ou aquela que mais se aproxime do seu desejo inicial).

Lembramos que a Lógica apenas aproxima a linguagem natural, e que as expressões do Cálculo Proposicional clássico constituem uma possível tradução em linguagem padronizada da leitura do usuário para o texto em estudo. Estas observações são particularmente importantes quanto à operação lógica da implicação e quanto ao significado do conectivo ou (as operações lógicas podem ser estudadas em 2, 14, 19, 27, 32, 33); podemos mesmo afirmar que muitos desenvolvi- mentos mais recentes da Lógica foram feitos para tentar resolver os problemas que surgem quando se interpretam certas operações, especialmente a operação implicação. Devido a estes problemas, foram criados diversos modelos alternativos, tais como: Lógicas Modal, Multivalorada, Paraconsistente, Difusa (13, 18, $22,30,31$ ).

Após a interpretação das frases em linguagem natural para expressões do Cálculo Proposicional clássico, estas últimas, para efeito de processamento, devem ser representadas por meio de expressões algébricas booleanas. Tal processo é trivial, unívoco, não apresentando dificuldades de monta.

A seguir vamos mostrar como o Cálculo Proposicional clássico pode ser equacionado em termos da Álgebra Booleana Binária $(3,12,17,26,33)$.

A Álgebra Booleana Binária é definida sobre um conjunto $A$ de dois elementos e apresenta três operações $\{+, \ldots$,$\} . Essas$ operações devem obedecer certas propriedades.

Denotando os dois elementos do conjunto $A$ com os símbolos 1 e 0 , temos: $A=\{1,0\}=$ conjunto formado pelos elementos 1 e 0 .

As operações,.+ , ' sobre $\{1,0\}$ obedecem às seguintes tabelas ( $x$ e $y$ representam elementos de $A$, e podem tomar os valores 1 ou 0 ):

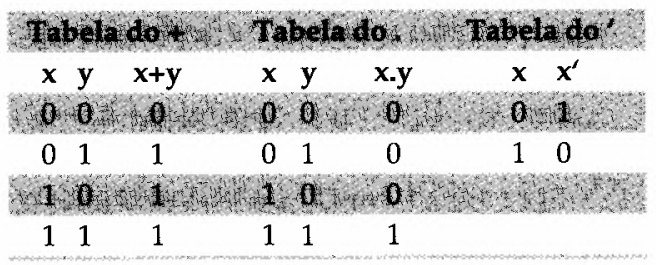

Todas as possíveis combinações de 0 e 1 (valores de $\mathrm{x}$ e y) estão presentes nestas tabelas.

O Cálculo Proposicional (ou Sentencial) é estudado em detalhes em $(14,19$, $27,32)$. Nele se estabelecem relações entre proposições. Estas são afirmações, não-contraditórias, que podem ser falsas ou verdadeiras (Observe-se que estas exigências referem-se apenas ao Cálculo Proposicional clássico, pois, no Cálculo Proposicional Paraconsistente, podem 
existir afirmações contraditórias; e, no Cálculo Proposicional Multivalorado, podem existir afirmações que não são nem verdadeiras, nem falsas; sugerimos que métodos similares aos aqui apresentados sejam estendidos a outros tipos de lógicas.).

As proposições podem ser relacionadas, ou operadas, por meio de conectivos lógicos do tipo NÃO, E, E/OU, IMPLICA, EQUIVALE. As expressões resultantes dessas operações admitem também um valor falso ou verdadeiro, determinado segundo regras vistas a seguir, e são chamadas proposições compostas (Por razões detalhadas adiante, o conectivo "ou inclusivo" do Cálculo Proposicional, usualmente grafado OU, será por nós escrito E/OU).

O Cálculo Proposicional clássico pode ser equacionado em termos da Álgebra Booleana Binária, definindo-se A como o conjunto formado pelos elementos "proposição verdadeira $V$ " e "proposição falsa $F^{\prime \prime}$, ou seja, $\mathbf{A}=\{\mathrm{V}, \mathrm{F}\}$

Desse modo, fazendo as correspondências indicadas na tabela a seguir:

$$
\begin{aligned}
& \mathrm{V} \leftrightarrow 1 \\
& \mathrm{~F} \leftrightarrow 0 \\
& \text { Operação E/OU sobre }\{\mathrm{V}, \mathrm{F}\} \leftrightarrow \\
& \text { operação + sobre }\{1,0\} \\
& \text { Operação E sobre }\{\mathrm{V}, \mathrm{F}\} \leftrightarrow \\
& \text { operação . sobre }\{1,0\} \\
& \text { Operação NÃO sobre }\{\mathrm{V}, \mathrm{F}\} \leftrightarrow \\
& \text { operação ' sobre }\{1,0\}
\end{aligned}
$$

obtemos uma Álgebra Booleana Binária. As tabelas da soma (+), do produto (.) e da complementação (') podem então ser reescritas em termos de $\mathrm{V}$ e $\mathrm{F}$, e serão, respectivamente, as tabelas que representam as operações E/OU, E e NÃO ( $x, y$ são proposições):

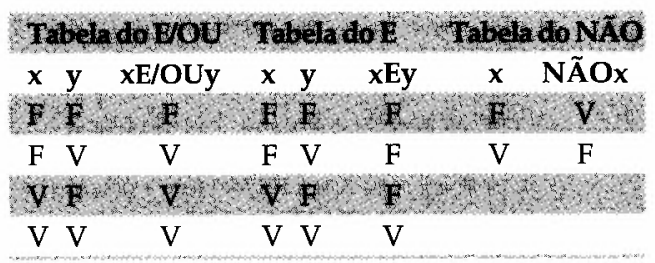

Por conveniência, de agora em diante, vamos usar indiferentemente os símbolos
V, F, E/OU, E, NÃO ou $1,0+, .,{ }^{\prime}$. Desse modo:

$z=x E / O U ~ y=x+y=$ disjunção de $x$ com y (soma booleana)

$w=x E y=x \cdot y=$ conjunção de $x \operatorname{com} y$ (produto booleano)

$\mathbf{v}=\mathrm{NA} O \mathrm{x}=\mathrm{x}^{\prime}=$ negação de $\mathrm{x}$ (complementação booleana)

$\mathbf{z}, \mathbf{w}, \mathbf{v}$ são proposições compostas.

Note-se que:

Se uma das proposições for verdadeira, a disjunção será verdadeira.

Se as duas proposições forem verdadeiras, a conjunção será verdadeira.

Substituindo as proposições básicas (também chamadas átomos) por identificadores (letras) e os conectivos por símbolos (tais como,$+ \ldots$, ), podemos obter expressões algébricas que simbolizam a proposição composta.

Por exemplo, a proposição composta:

"Não houve aula hoje, ou o ônibus atrasou e a passagem está cara", após ser interpretada no Cálculo Proposicional clássico, seria traduzida pela seguinte expressão algébrica:

$$
\begin{aligned}
& \mathbf{a}^{\mathbf{\prime}}+\mathbf{( b . c )} \\
& \text { com as proposições básicas: } \\
& \mathbf{a}=\text { houve aula hoje } \\
& \mathbf{b}=\text { o ônibus atrasou } \\
& \mathrm{c}=\mathrm{a} \text { passagem está cara }
\end{aligned}
$$

A seguir, é fornecida uma lista de proposições compostas e sua tradução em expressões algébricas da Álgebra Booleana Binária. Repetimos que nem sempre a tradução do texto é relativamente simples, como no exemplo acima, devido às ambigüidades da linguagem natural.

A tabela 1 apresenta uma lista de traduções $(21,33)$ de expressões do Cálculo Proposicional (em língua portuguesa) para expressões algébricas. Nestas expressões comparecem variáveis literais $(x, y$, $z, \ldots)$ e as operações $(+, \ldots, ')$. Apresentamos também alguns símbolos $(\rightarrow,=, 1, \oplus)$ empregados quando se desejam expressões mais compactas. Note-se que, muitas vezes, escreveremos abc com o significando de a.b.c. 


\section{Tabela 1: Tradução de expressões padronizadas do Cálculo Proposicional clássico para expressões álgébricas}

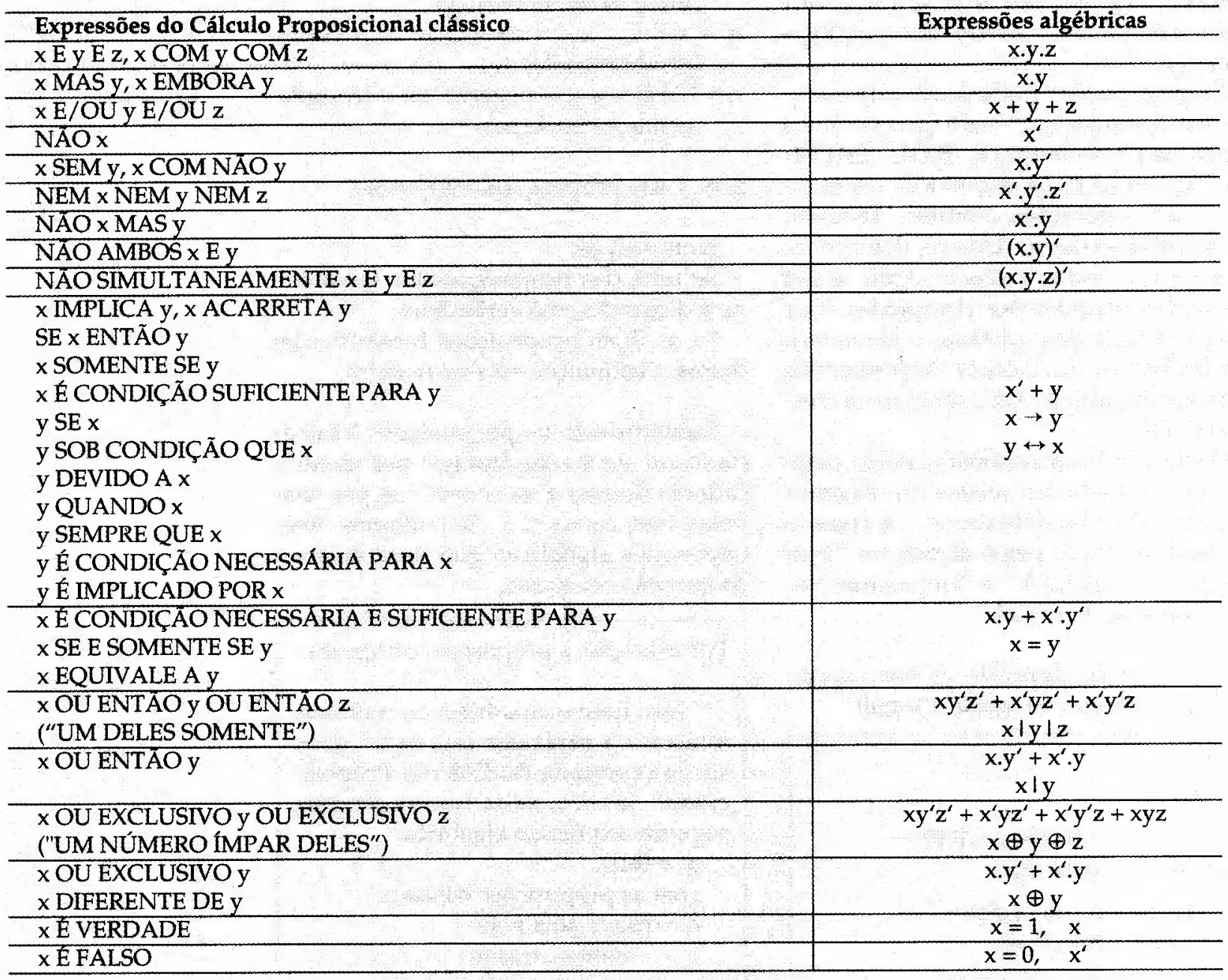

Algumas observações sobre a tabela 1 são oportunas:

I - O conectivo OU ENTĀO é uma proposta nova, merecendo um estudo à parte:

Na Álgebra Booleana, existem as operações $+(\mathrm{OU}) \mathrm{e} \oplus$ (OU EXCLUSIVO ou OUX), que obedecem às tabelas:

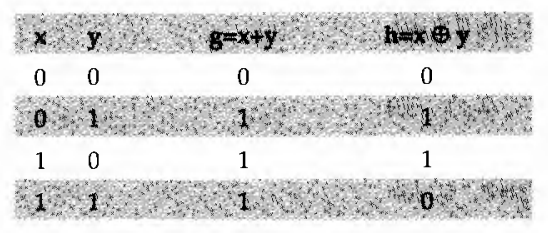

Assim, $\mathrm{g}=\mathrm{x}$ OU y será 1 , se $\mathrm{x}, \mathrm{ou} \mathrm{y}$, ou ambos forem iguais a 1 ; $\mathrm{e} h=x$ OUX y será 1 , se $x$ ou y (mas não ambos) forem iguais a 1 . O conectivo OU recebe às vezes o nome de OU INCLUSIVO, em contraposição ao OU EXCLUSIVO. Adiante, veremos que $\mathrm{h}=\mathrm{x} \oplus \mathrm{y}$ pode ser escrito, em termos das operações $t, .,{ }^{\prime}$, como $h=x \cdot y^{\prime}+x^{\prime} y$.

Na linguagem natural, a conjunção OU apresenta diversos significados, como por exemplo:

a. Em "Dou desconto a professores, ou a engenheiros, ou a idosos", entende-se que, pertencendo a uma só das categorias, ou a qualquer combinação duas a duas delas, ou às três, o desconto estará garantido.

b. Em "Hoje à noite vou ao teatro, ou ao cinema, ou ao estádio", entende-se que hoje à noite irei a um só dos lugares citados.
Na Lógica Simbólica, à qual pertence o Cálculo Proposicional clássico, adotou-se para a operação OU o sentido do item a acima, ou seja, o sentido do OU INCLUSIVO da álgebra Booleana (32).

A operação OU EXCLUSIVO, quando aplicada a apenas dois elementos, traduz convenientemente o sentido do ou do item b acima, ou seja, o sentido de "um deles somente". Com efeito, $h=x \oplus y=x y^{\prime}+x^{\prime} y$ indica que $h=1$ se $x$ $=1$, ou $\mathrm{y}=1$, não podendo serem ambos iguais a 1. Porém, quando aplicada a mais de dois elementos, como em $\mathrm{p}=\mathrm{x} \oplus \mathrm{y} \oplus \mathrm{z}=\mathrm{x} \oplus(\mathrm{y} \oplus \mathrm{z})=$ $(\mathrm{x} \oplus \mathrm{y}) \oplus \mathrm{z}$ (o OU EXCLUSIVO tem a propriedade da associatividade), obtém-se, por manipulação algébrica $(2,17,26,33)$, que $\mathrm{p}=x y^{\prime} z^{\prime}+$ $x^{\prime} y z^{\prime}+x^{\prime} y^{\prime} z+x y z$. Ou seja, $p=1$ se $x=1$, ou $y=$ 1 , ou $z=1$, ou 
$x=y=z=1$, não podendo dois deles serem simultaneamente iguais a 1 .

Generalizando, a aplicação da operação OU EXCLUSIVO a n elementos levará à conclusão de que havendo um número Ímpar de elementos iguais a 1 , o resultado será 1.

Esta conclusão mostra que esta operação é inadequada para traduzir o sentido do ou do item $b$ acima, pois, na linguagem natural, quando falamos que "vamos ao lugar $x$, ou ao $y$, ou ao $z^{\prime \prime}$, nunca queremos dizer que vamos a um número ímpar deles!

Para traduzir o sentido do ou do item b (o sentido de "um deles somente"), muito comum na linguagem natural, convém definirmos o conectivo OU ENTÃO (símbolo I) pelas expressōes: $\mathrm{q}=\mathrm{x}$ OU ENTÃO $\mathrm{y}=\mathrm{x} \mid \mathrm{y}=\mathrm{xy}^{\prime}+$ $x^{\prime} y$

$$
r=x \text { OU ENTÃO y OU ENTÃO } z=
$$$$
x|y| z=x y^{\prime} z^{\prime}+x^{\prime} y z^{\prime}+x^{\prime} y^{\prime} z
$$

$\mathbf{s}=\ldots=x|y| z|\ldots| w=x y^{\prime} z^{\prime} \ldots$ $w^{\prime}+x^{\prime} y z^{\prime} \ldots w^{\prime}+x^{\prime} y^{\prime} z . . w^{\prime}+\ldots$ $+x^{\prime} y^{\prime} z^{\prime} . . w$

Desse modo, $q, r, \ldots s$ serão iguais a 1 , se e somente se um dos elementos operados for igual a 1 .

Para maior clareza, o ou do item a (o OU INCLUSIVO) será aqui grafado $\mathrm{E} / \mathrm{OU}$.

Em resumo, haverá três tipos de ou:

a) E/OU, com o significado de "OU INCLUSIVO“(símbolo + ).

Assim: $g=x+y+z+\ldots+w$ sera igual a 1 , desde que pelo menos um dos operandos seja igual a 1 (será igual a zero só se todos os operandos forem iguais a zero).

b) OU ENTÃO, com o significado de "UM DELES SOMENTE"(símbolo I).

Assim: $\mathrm{s}=\mathrm{x}|\mathrm{y}| \mathrm{z}|\ldots| \mathrm{w}$ será igual a 1 , se e somente se um dos elementos operados for igual a 1 .

c) OU EXCLUSIVO, com o significado de "UM NÚMERO ÍMPAR DELES"(símbolo $\oplus$ ).

Em geral, este conectivo não é usado na linguagem, mas é importante na Álgebra Booleana aplicada a circuitos lógicos (em codificação, filtros etc.)
Assim: $\mathrm{p}=\mathrm{x} \oplus \mathrm{y} \oplus \mathrm{z} \oplus \ldots \oplus \mathrm{w}$ será igual a 1 , se e somente se um número ímpar de elementos operados for igual a 1 .

A tabela 2 apresenta as três operações acima descritas, para quatro operandos.

Notas:

1) Se $x, y, w, z$ forem mutuamente exclusivos, as operações $\mathrm{OU}$ ENTÃO e E/OU levam ao mesmo resultado:

$x|y| z \mid w=x+y+z+w$

2) Para dois elementos, OU ENTÃO e OU EXCLUSIVO levam ao mesmo resultado: $x \mid y=x \oplus y=x y^{\prime}+x^{\prime} y$ (ou $x$, ou $y$, mas não ambos)

3) Embora para a operaçăo OU ENTÃO seja válida a igualdade: $(x \mid y)|z=x|(y \mid z)$ o resultado é diferente de $x|y| z=x y^{\prime} z^{\prime}+x^{\prime} y z^{\prime}+x^{\prime} y^{\prime} z$

Portanto, quando se utiliza o conectivo OU ENTÃO, ele não pode ser aplicado repetidamente, adicionando-se um elemento de cada vez, mas deve ser aplicado em bloco, utilizando-se as expressões empregadas em sua definição. Diversamente dos operadores E/OU, E, OU EXCLUSIVO etc. que são diádicos (operam dois elementos de cada vez), o OU ENTÃO é um operador n-ádico.

4) Do mesmo modo que na utilização da função IMPLICAÇÃO (2 $14,32,33$ ), certos cuidados de vem ser tomados quando se emprega o conectivo OU ENTÃO na tradução de frases da linguagem para expressões do Cálculo Proposicional clássico (ou para expressões algébricas), pois, embora corretos (ou seja, decorrentes da definição), certos resultados, num primeiro exame, podem contrariar a intuição. Por exemplo, as seguintes expressões são válidas (veja a tabela 1 para entendimento dos símbolos):

$\mathrm{a} \mid \mathrm{a}=\mathrm{a} \oplus \mathrm{a}=0$

a $\mid(a \cdot b)=a \oplus(a \cdot b)=a \cdot b^{\prime}$

$a \mid(a+b)=a \oplus(a+b)=a^{\prime} \cdot b$

$a|b|(a \cdot b)=a \cdot b^{\prime}=a^{\prime}+b=a \mid b$

$a|b|(a+b)=0$

Para verificar o quanto estes resultados contrariam a intuição num primeiro momento, basta se fazerem as substituições:

$$
\begin{aligned}
& \mathrm{a}=\text { ir ao teatro } \\
& \mathrm{b}=\text { ir ao cinema } \\
& +=\mathrm{E} / \mathrm{OU} \\
& =\mathrm{E} \\
& \mid=\text { OU ENTÃO } \\
& \oplus=\text { OU EXCLUSIVO } \\
& 0=\text { FALSO }
\end{aligned}
$$

II - Prova-se que são válidas as seguintes igualdades:

$\mathrm{x}$ OU EXCLUSIVO $\mathrm{y}=\mathrm{x} \oplus \mathrm{y}=\mathrm{xy}^{\prime}$ $+x^{\prime} y=(x$ E NÃO y) E/OU (NÃO x E y)

$x$ IMPLICA $y=x \rightarrow y=x^{\prime}+y=$ $\mathrm{NÃO} \times \mathrm{E} / \mathrm{OU} y$

$x$ EQUIVALE $A y=(x=y)=x y+$ $x^{\prime} y^{\prime}=(x$ E y) E/OU (NÃO $x e$ $\mathrm{N} \tilde{\mathrm{A} O} \mathrm{y}$ )

III - Conforme foi citado, em nosso contexto, as ambigüidades presentes na linguagem natural devem ser eliminadas antes de se traduzirem as frases em expressões do Cálculo Proposicional clássico (ou expressões algébricas).

Como exemplo de ambigüidade, o mesmo enunciado em português : "Ir ao teatro $(x)$ e ir ao cinema (y) ou ir ao estádio (z)" pode levar a várias expressões diferentes, conforme a intepretação: $(x, y)$ $+z, x \cdot(y+z),(x \cdot y) \mid z, x \cdot(y \mid z) \cdot \mathrm{Ou}-$ tro exemplo de possíveis interpretações diversas, conforme o contexto, é o seguinte(11):

A frase: "Andar de bicicleta e a cavalo" poderia ser interpretada no Cálculo Proposicional clássico, como:

- Andar de bicicleta E andar a cavalo (contexto: um artista de circo);

- Andar de bicicleta E/OU andar a cavalo (contexto: durante o dia);

- Andar de bicicleta OU ENTÃO andar a cavalo (contexto: num certo instante):

- Andar de bicicleta IMPLICA andar a cavalo (contexto: necessita-se da bicicleta para se chegar ao cavalo e montá-lo);

- Andar de bicicleta EQUIVALE A andar a cavalo (contexto: para ir a um certo local, pode-se utilizar um ou outro, equivalentemente).

As ambigüidades podem ser resolvidas pelo usuário, baseandose na intenção, no sentido das frases, no bom senso e no conhecimento do contexto no qual o problema está inserido. Isto vale igualmente para se determinar qual o significado dos conectivos ou utilizados. 
Tabela 2: Operações OU, OU ENTÃo, OU EXCLUSIVO para quatro variáveis

\begin{tabular}{cccccclc}
\hline $\mathbf{i}$ & $\mathbf{x}$ & $\mathbf{y}$ & $\mathbf{z}$ & $\mathbf{w}$ & $\mathbf{g}=\mathbf{x}+\mathbf{y}+\mathbf{z}+\mathbf{w}$ & $\mathbf{s}=\mathbf{x}|\mathbf{y}| \mathbf{z} \mid \mathbf{w}$ & $\mathbf{p}=\mathbf{x} \oplus \mathbf{y} \oplus \mathbf{z} \oplus \mathbf{w}$ \\
\hline 0 & 0 & 0 & 0 & 0 & 0 & 0 & 0 \\
1 & 0 & 0 & 0 & 1 & 1 & $1\left(\mathrm{i}=1=2^{0}\right)$ & 1 \\
2 & 0 & 0 & 1 & 0 & 1 & $1\left(\mathrm{i}=2=2^{1}\right)$ & 1 \\
3 & 0 & 0 & 1 & 1 & 1 & 0 & 0 \\
4 & 0 & 1 & 0 & 0 & 1 & $1\left(\mathrm{i}=4=2^{2}\right)$ & 1 \\
5 & 0 & 1 & 0 & 1 & 1 & 0 & 0 \\
6 & 0 & 1 & 1 & 0 & 1 & 0 & 0 \\
7 & 0 & 1 & 1 & 1 & 1 & 0 & 1 \\
8 & 1 & 0 & 0 & 0 & 1 & $1\left(\mathrm{i}=8=2^{3}\right)$ & 1 \\
9 & 1 & 0 & 0 & 1 & 1 & 0 & 0 \\
10 & 1 & 0 & 1 & 0 & 1 & 0 & 0 \\
11 & 1 & 0 & 1 & 1 & 1 & 0 & 0 \\
12 & 1 & 1 & 0 & 0 & 1 & 0 & 1 \\
13 & 1 & 1 & 0 & 1 & 1 & 0 & 0 \\
14 & 1 & 1 & 1 & 0 & 1 & 0 & 1 \\
15 & 1 & 1 & 1 & 1 & 1 & 0 &
\end{tabular}

\section{FUNÇס̃ES BOOLEANAS}

Antes de resolver o problema apresentado na seção 1 , necessitamos de mais alguns conceitos.

Função booleana de $\mathrm{N}$ variáveis $\mathrm{a}, \mathrm{b}, \mathrm{c}$, ..., $\mathrm{n}$ é uma correspondência entre cada uma das possíveis combinações de valores 0, 1 destas variáveis, com um e um só dos valores 0 ou 1. Esta correspondência pode ser apresentada por meio da "tabela de combinações", vista a seguir.

Para $N=3$ variáveis, uma possível função de $a, b, c$ é a função $z$, especificada pela tabela seguinte (as combinações abc são dispostas em ordem de 000 a 111).

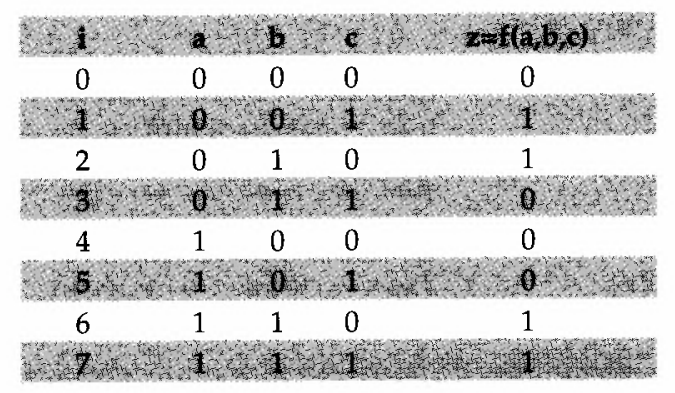

A seqüência $J=11000110$ (que é $a$ coluna da direita lida no sentido do maior $i$ para o menor, ou seja, de baixo para cima), desde que se especifique a ordem das variáveis, define completamente a função $z$. Esta seqüência, junto com $\mathrm{N}$ (número de variáveis), e as variáveis abc, na ordem utilizada na tabela, recebe o nome de Transformada Numérica (TN), e foi a utilização deste conceito que possibilitou a dedução do Algoritmo RQ (A proposta da TN encontra-se em 23, 24 e sua formalização em $4,5,11$ ).

Um modo usual de representar uma função booleana é a notação de Caldwell, que especifica as posições dos bits 1 na seqüência J.

No exemplo dado temos bits 1 nas posições $7,6,2$, 1. Na notação de Caldwell escreveríamos:

$$
z=\sum(7,6,2,1)
$$

Observe-se que, da notação de Caldwell, podemos facilmente determinar $\mathrm{J}$ e, tendo $\mathrm{J}$, podemos determinar facilmente a notação de Caldwell da função. 
Outro modo usual de representar uma função é a forma algébrica; neste caso, a função acima seria escrita:

$$
\begin{aligned}
z & =a^{\prime} \cdot b^{\prime} \cdot c+a^{\prime} \cdot b \cdot c^{\prime}+a \cdot b \cdot c^{\prime}+a \cdot b \cdot c \\
& =b \cdot c^{\prime}+a \cdot b+a^{\prime} b^{\prime} \cdot c
\end{aligned}
$$

A primeira expressão é obtida diretamente da tabela e a segunda é deduzida da primeira por manuseio algébrico (2, 17, 26, 33).

Atribuindo a a, b, c os valores da tabela de combinações, linha por linha, e efetuando as operações de acordo com as tabelas de,.,+ ', obtemos a coluna $z$.

Passar da notação algébrica de $z$ para J chama-se "transformar $z$ " e passar de J para a notação algébrica denomina-se "antitransformar J", e há métodos próprios paras as duas operações(11). Notese que J depende da ordem em que se colocam as variáveis $a, b, c, .$. na tabela de combinações.

As igualdades vistas anteriormente, envolvendo as operações $\oplus$ (OU EXCLUSIVO), $\rightarrow$ (IMPLICA) e = (EQUIVALE), são obtidas representando algebricamente estas funções a partir de suas tabelas de combinações.

$\begin{array}{ccccc}\text { a } & b & a \oplus b & a b & a=b \\ 0 & 0 & 0 & 1 & 1 \\ 0 & 1 & 1 & 1 & 0 \\ 1 & 0 & 1 & 0 & 0 \\ 1 & 1 & 0 & 1 & 1\end{array}$

\section{O PROBLEMA DA DECISÃO QUALITATIVA}

O Problema da Decisão Qualitativa no Cálculo Proposicional (PDQ) consiste no seguinte: dados um Enunciado En (sistema de expressões do Cálculo Proposicional clássico) e uma Questão $Q$ (função booleana de algumas variáveis de En), determinar a Resposta R (função booleana de algumas ou todas as variáveis de En que não comparecem em $Q$ ), tal que $($ En. $R) \rightarrow Q)=1$ ou, equivalentemente, $(E n \rightarrow(R \rightarrow Q)=1$. Ou seja: queremos determinar $R$, tal que, se En e $R$ forem verdadeiros, então $Q$ também será verdadèira (ou também, se En for verdadeiro então R implica Q). Dado um conjunto de relações não quantificadas entre eventos (proposições), sua resolução permite a obtenção de outras relações entre proposições escolhidas sendo estas selecionadas dentre aquelas proposições originalmente fornecidas. Em particular, a partir do citado conjunto de relações, o PDQ é útil para resolver problemas lógicos do tipo: como agir em relação a certas proposições escolhidas, para forçar a ocorrência de uma situação desejada envolvendo outras proposições.

Em $(1,11)$ encontram-se detalhes sobre o Problema da Decisão Qualitativa, além dos problemas da "Obtenção de todas as teses possíveis"e da "Demonstração de teoremas" no Cálculo Proposicional, com sugestões de extensão para o Cálculo de Predicados. Aqui, mostraremos apenas como resolver o PDQ a partir da resolução de equações booleanas, nossos desenvolvimentos tendo enfoque operacional, sem preocupação de rigor.

O Problema da Decisão especifica um Enunciado En, função de $\mathrm{N}$ variáveis. Inicialmente, a Questão $Q$ é especificada como função de algumas dessas variáveis (variáveis dependentes), e a Resposta $R$, como função de um subconjunto das variáveis remanescentes (variáveis independentes). No caso de existirem, no problema proposto, variáveis ausentes - que não foram especificadas nem na resposta $R$, nem na Questão Q-, elas são adotadas como variáveis da Questão $Q$ (funcionam como variáveis brancas, pois $Q$ não depende dessas variáveis).

Nosso método de resolução, primeiramente, transforma o PDQ proposto em expressões algébricas, constituindo um sistema simultâneo de equações booleanas. Como qualquer sistema deste tipo pode ser transformado em uma equação equivalente, os algoritmos para resolução destes sistemas, sem perda de generalidade, são desenvolvidos para a resolução de equações equivalentes.

A seguir, a equação equivalente do Enunciado é determinada com as $\mathrm{N}$ va- 
Nosso método de resolução, primeiramente, transforma o PDQ proposto em expressões algébricas, constituindo um sistema simultâneo de equações booleanas. riáveis em ordem tal que aquelas que comparecem em $R$ (as independentes) estejam à direita (veja a seção seguinte). Desse modo, teremos:

$w\left(x_{1}, \ldots, x_{i}, x_{j+1}, \ldots, x_{N}\right)=$ 1 é a equação equivalente do Enunciado;

$R=R\left(x_{j+1}, \ldots, x_{N}\right)$ é função de $\mathrm{N}$ - j variáveis independentes;

$Q=Q\left(x_{1}, \ldots, x_{i}\right)$ é função de $j$ variáveis dependentes.

Após isso, para resolver o PDQ, fazemos o seguinte;

1) Resolvemos a equação equivalente, obtendo as variáveis dependentes $x_{1}, \ldots, x_{i}$ (todas elas, mesmo as inicialmente ausentes em $Q$ ) em função das variáveis independentes $x_{j+1}, \ldots, x_{N}$.

2) Substituimos as expressões das variáveis dependentes $\left(x_{1}, \ldots x_{i}\right)$, obtidas em função das independentes $\left(x_{j+1}, \ldots, x_{N}\right)$, na função $Q=Q\left(x_{1}, \ldots, x_{i}\right)$. Evidentemente, utilizamos apenas as expressões das variáveis dependentes das quais $Q$ é função. Se obtivermos uma só expressão, esta será $R$; se existirem diversas soluções, devemos efetuar o produto booleano entre elas (para maiores detalhes, veja 11).

\section{RESOLUÇÃO DO PROBLEMA}

Como primeiro passo, vamos escrever o sistema de equações que traduz o problema proposto e transformá-lo numa só equação equivalente ao sistema.

Adotando as proposições:

$\mathrm{A}=$ Aperfeiçoar o produto

$\mathrm{B}=$ Gastar muito em propaganda

$\mathrm{C}=$ Pagar altas comissões

$\mathrm{D}=$ Elevar o preço de venda

$\mathrm{E}=$ Ter grande volume de vendas

$\mathrm{F}=$ Ter alto lucro o leitor poderá verificar, consultando a seção 1, que as cinco afirmações ("verdades") do Enunciado podem ser escritas:

$$
\begin{aligned}
& \text { I) }\left(\mathrm{A}+\mathrm{B}^{\prime}+\mathrm{C}+\mathrm{D}\right) . \mathrm{E}^{\prime} \rightarrow \mathrm{F}^{\prime} \\
& \text { II) }(\text { B.A' }) \mid(\text { A.B' }) \rightarrow(\text { E.F })^{\prime} \\
& \text { III) } \mathrm{F}+\mathrm{E} \rightarrow\left(\mathrm{D}^{\prime} . \mathrm{B}\right) \mid\left(\mathrm{D}^{\prime} . \mathrm{B}^{\prime} . \mathrm{A} \cdot \mathrm{C}^{\prime}\right) \\
& \text { IV) }\left(D^{\prime} . B . C\right) \mid\left(D^{\prime} . C^{\prime} . A\right) \rightarrow E \\
& \text { V) B.A.D'.E } \rightarrow \text { F }
\end{aligned}
$$

Como sabemos, $x \rightarrow y$ (implicação) é equivalente $a x^{\prime}+y$. Como as afirmações do problema são admitidas verdadeiras (base do problema), escreveremos $x^{\prime}+y$ $=1$. Aplicando essa igualdade às implicações citadas, por manuseio das expressões, obtemos:

$$
\begin{aligned}
& \text { I) } E+A^{\prime} \cdot B \cdot C^{\prime} \cdot D^{\prime}+F^{\prime}=1 \\
& \text { II) } A^{\prime} \cdot B^{\prime}+A \cdot B+E^{\prime}+F^{\prime}=1 \\
& \text { III) } E^{\prime} \cdot F^{\prime}+B \cdot D^{\prime}+A \cdot B^{\prime} \cdot C^{\prime} \cdot D^{\prime}=1 \\
& \text { IV) } D+A^{\prime} \cdot C^{\prime}+B^{\prime} \cdot C+E=1 \\
& \text { V) } A^{\prime}+B^{\prime}+D+E^{\prime}+F=1
\end{aligned}
$$

Se dispomos de várias expressões iguais a 1, a expressão obtida pelo produto booleano de todas as expressões entre si, igualada a 1 , é equivalente às expressões originais dadas.

Assim, chamando de $\mathrm{P}, \mathrm{Q}, \mathrm{R}, \mathrm{S}, \mathrm{T}$ às expressões à esquerda nas igualdades $I$, II, III, IV, V, respectivamente, temos:

\section{P.Q.R.S.T $=1$}

Efetuando o produto obtemos uma função de E, F, A, B, C, D. que chamaremos de $w(E, F, A, B, C, D)$.

Portanto, $\mathrm{w}(\mathrm{E}, \mathrm{F}, \mathrm{A}, \mathrm{B}, \mathrm{C}, \mathrm{D})=$ P.Q.R.S.T $=1$

A ordem das variáveis foi escolhida 


\section{APLICAÇÃO DO ALGORITMO RQ}

0 algoritmo

Resto-Quociente

é facilmente

programável em

computadores.

tendo em conta que o problema solicita as variáveis $\mathrm{E}, \mathrm{F}$ em função das variáveis A, B, C, D.

A função $w$ é chamada função equivalente ao sistema e pode ser expressa na notação de Caldwell. Em geral, o produto pode ser mais facilmente efetuado com auxílio da Transformada Numérica (TN); obtém-se A TN de w e, a partir da mesma, obtém-se a notação de Caldwell de w.

No exemplo dado, efetuando o produto obtemos, para a equação equivalente:

$\mathrm{w}(\mathrm{E}, \mathrm{F}, \mathrm{A}, \mathrm{B}, \mathrm{C}, \mathrm{D})=\sum(62,60,40,38,36,20$, $15,13,11,10,9,7,5,4,3,2,1,0$ )

Os detalhes desta multiplicação serão omitidos aqui, por não serem essenciais à compreensão do método (veja 11).

Ainda, o problema pede, conforme a seção 1:

a) F em função de A, B, C, D

(Questão Q1)

b) E em função de A, B, C, D

(Questão Q2)

c) E.F em função de A, B, C, D

(Questão Q3)

Em seguida, vamos determinar os itens a, b e c de acordo com o roteiro da seção 4. Para a resolução da equação equivalente utilizaremos o Algoritmo Resto-Quociente, ou Algoritmo RQ $(6,10,11)$.
Apresentaremos o algoritmo à medida que o aplicarmos na resolução do problema proposto.

No problema temos $\mathrm{N}=6$ variáveis $\mathrm{e}$ desejamos duas dessas variáveis ( $\mathrm{E}$ e $\mathrm{F}$ ) em função de outras 4 (portanto há 2 variáveis dependentes e 4 independentes). Chamando de $\mathrm{j}$ o número de variáveis dependentes, temos:

I) Dividem-se os números da notação de Caldwell de $w$ por $2^{\mathrm{N}-\mathrm{j}}=2^{4}=16$, obtendose quocientes q e restos $r$ :

\begin{tabular}{|c|c|c|c|c|c|}
\hline $\mathbf{m}$ & $q$ & $\mathbf{r}$ & $\mathbf{m}$ & $\mathbf{q}$ & $\mathbf{r}$ \\
\hline 62 & 3 & 14 & 10 & 0 & 10 \\
\hline 60 & 3 & 12 & 9 & 0 & 9 \\
\hline 40 & 2 & 8 & 7 & 0 & 7 \\
\hline 38 & 2 & 6 & 5 & 0 & 5 \\
\hline 36 & 2 & 4 & 4 & 0 & 4 \\
\hline 20 & 1 & 4 & 3 & 0 & 3 \\
\hline 15 & 0 & 15 & 2 & 0 & 2 \\
\hline 13 & 0 & 13 & 1 & 0 & 1 \\
\hline 11 & 0 & 11 & 0 & 0 & 0 \\
\hline
\end{tabular}

Por exemplo, $62 \div 16=3$, com resto 14 .

II) Ordenam-se os quocientes q de acordo com os restos $r$ correspondentes (r vai de $2^{\mathrm{N}-\mathrm{i}}-1=15$ a 0 ):

\begin{tabular}{|c|c|c|c|}
\hline $\mathbf{r}$ & $q$ & $\mathbf{r}$ & $q$ \\
\hline 15 & 0 & 7 & 0 \\
\hline 14 & 3 & 6 & 2 \\
\hline 13 & 0 & 5 & 0 \\
\hline 12 & 3 & 4 & $2,1,0$ \\
\hline 11 & 0 & 3 & 0 \\
\hline 10 & 0 & 2 & 0 \\
\hline 9 & 0 & 1 & 0 \\
\hline 8 & 2 & 0 & 0 \\
\hline
\end{tabular}


III) Determinam-se todas as combinações de quocientes $q$, tomando um só $q$ para cada $r$, na ordem $r=2^{\mathrm{N}-\mathrm{i}}-1$ a 0 (são as várias sequiências de números que se obtém, lendo-se a coluna da direita da tabela anterior de cima para baixo).

Resultam três soluções:

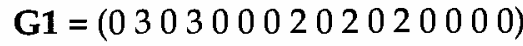

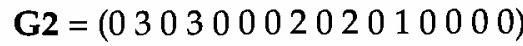

$\mathbf{G} 3=\left(\begin{array}{llll}0 & 303000202000000\end{array}\right)$

IV) As soluções transformadas são obtidas construindo-se, para cada Gi, uma matriz cujas colunas são os elementos de $\mathrm{Gi}$, expressos em base 2, em colunas escritas de cima para baixo.

\begin{tabular}{|c|c|}
\hline \multicolumn{2}{|c|}{ Solução 1) G1: } \\
\hline & E1: $\quad 0101000101010000$ \\
\hline & F1: 0101000000000000 \\
\hline Solução 2) G & G2: \\
\hline & E2: 0101000101000000 \\
\hline & F2: $\quad 0101000000010000$ \\
\hline Solução 3) G & G3: \\
\hline & E3: 0101000101000000 \\
\hline & F3: $\quad 0101000000000000$ \\
\hline
\end{tabular}

Por exemplo, na solução 1 , o segundo número é 3 , o qual em base 2 é 11 .

Para obter a Questão Q3, determinamos E.F nas três soluções encontradas; para isto, basta efetuar o produto booleano, bit a bit, nas três soluções acima, obtendo-se:

\section{Solução 1, 2 e 3: \\ E.F: 0101000000000000}

(solução única)

As Respostas R, conforme citado na seção 4, serão determinadas efetuando-se os produtos das diversas soluções entre si.
Desse modo, obtemos:

$\mathrm{Q} 1=\mathrm{F}, \quad \mathrm{R} 1=\mathrm{F} 1 \cdot \mathrm{F} 2 \cdot \mathrm{F} 3: 0101000000000000$

$\mathrm{Q} 2=\mathrm{E}, \quad \mathrm{R} 2=\mathrm{E} 1 \cdot \mathrm{E} 1 \cdot \mathrm{E} 3: 0101000101000000$

Q3 = E.F, R3 : 0101000000000000

Antitransformando (veja 11), obtemos as Respostas para as três Questões:

a) Questão Q1 = F

Resposta R1 = A.B.D

b) Questão Q2 = E

Resposta $\mathrm{R} 2=\mathrm{A} \cdot \mathrm{C}^{\prime} \cdot \mathrm{D}^{\prime}+\mathrm{B} \cdot \mathrm{C} \cdot \mathrm{D}^{\prime}+\mathrm{A} \cdot \mathrm{B} \cdot \mathrm{D}^{\prime}$

c) Questão Q3 = E.F

Resposta R3 = A.B.D

\section{INTERPRETAÇÃO DOS RESULTADOS}

As Respostas são obtidas "vertendo" as expressões algébricas dos $\mathrm{Ri}$, utilizando a tabela das traduções da seção 2 e substituindo cada letra pela proposição correspondente.

\section{Obtemos:}

a) Para Ter alto lucro, deve-se Aperfeiçoar o produto E Gastar muito em propaganda E NÃO Elevar o preço de venda.

b) Para Ter grande volume de vendas, deve-se Aperfeiçoar o produto E NÃO Pagar altas comissões E NÃO Elevar o preço de venda E/OU Gastar muito em propaganda E Pagar altas comissões E NẪO Elevar o preço de venda E/OU Aperfeiçoar o produto E Gastar muito em propaganda E NÃO Elevar o preço de venda.

c) Para Ter grande volume de vendas E Ter alto lucro, deve-se Aperfeiçoar o produto E Gastar muito em propaganda E NÃO Elevar o preço de venda.

Algumas considerações podem ser feitas sobre as soluções determinadas:

As Respostas $\mathrm{R}$ obtidas para as três Questões (E, F, E.F), resolvem o Problema de Decisão Qualitativa nos três casos: 


$$
\mathrm{En} \rightarrow(\mathrm{R} \rightarrow \mathrm{Q})=1
$$

Adotando En como verdadeiro, se for assegurado, em cada Resposta, que $\mathrm{R}=1$, teremos, conseqüentemente, $Q=1$.

Nas respostas $R$, vemos que cada monômio das expressões constitui uma alternativa para se obter a respectiva Resposta igual a 1. Por exemplo, na Resposta para Q2 = E temos três monômios, e, portanto, três possíveis alternativas:

$A=1, C=\varnothing, D=0$ (com B qualquer) $\rightarrow R 2=1$ $\mathrm{B}=1, \mathrm{C}=1, \mathrm{D}=0$ (com $\mathrm{A}$ qualquer) $\rightarrow \mathrm{R} 2=1$ $\mathrm{A}=1, \mathrm{~B}=1, \mathrm{D}=0$ (com $\mathrm{C}$ qualquer $) \rightarrow \mathrm{R} 2=1$

Desse modo, do exame das expressões das Respostas, podemos deduzir quais os valores que devemos atribuir a $A, B, C, D$ para obtermos, em cada problema, $\mathrm{R}=1$ e, portanto, $Q=1$.

No caso de o usuário desejar formular outra Questão sobre o mesmo Enunciado, deverá fazê-lo antes da resolução da equação equivalente. Por exemplo, se o problema especificasse como Questão (já em linguagem algébrica):

$$
\mathrm{Q} 4=\mathrm{A}^{\prime} \cdot \mathrm{E}+\mathrm{A} \cdot \mathrm{E}^{\prime}
$$

com a Resposta em função de C, D, F, a equação equivalente seria resolvida determinando $(A, E, B)=f(C, D, F)$.

\section{BIBLIOGRAFIA}

1. BRUNAZO FILHO, A., DEL PICCHIA, W. Reșolução de problemas de decisāo qualitativa no cálculo oroposicional ufilizando equaçōes booleanas. São Paulo, Departamento de Engenharia de Eletricidade da Escola Politécnica da USP, abr. 1988, 510.

2. CHINAL, J. Techniques boolếenes et calculateurs arithmétiques. Parls: Dunod, 1967.

3. DAGHLIAN, J. Lógica $\theta$ algebra de Boole. Sẵo Paulo: Atlas, 1986.

4. DEL PICCHIA, W. A transformada numérica e sua aplicação à simplificação de funçōes e à resolução de equaçóes booleanas. Sāo Paulo, mar. 1971164 p. Tese (Livre Docência) - Escola Politécnica, Universidade de São Pâulo.

5. DEL PICCHIA, W.; MARTINS, W.W. The numerical transform. São Paulo, Departamento de Engenharia de Eletricidade da Escola Politécnica da USP, mar. 1974, 3 v. (Publicaçōes Internas, n. 16 - Basis. 41 p., п. 17 - Simplificafion of Boolean functions, $51 \mathrm{p}$. ก. 18 - Resolution of Boolean equations, $27 \mathrm{p.}$.)

6. DEL PICCHIA, W. A simple and fast algarithm for the resolution of Boolean squations. São Paulo, Departamento de Engenharia de Eletricidade da Escola Politécnica da USP, mar.1974. $18 \mathrm{p}$. (Publicaçāo Interna, n. 19).

7. Um algoritmo numérico para o manuseio de funçóes booleanas; o probiema da substituição. São Paulo, Departamento de Engenharia de Eletrici: dade da Escola Politécrica da USP, mar. 1974, 8 p. (Publicação Interna, n. 20).

8. Sistematizacão do projeto de máquinas seqüenciais, para diferentes elementos de realimentaçắo. Săo Paulo, Departamento de Engentharia de Eletricidade dà Escola Politécnica da USP, mar. 1974, 15p. (Publicaçāo Interna, n. 21).

9. Resoluçãa de equaçōes booleanas; aplicaçāo a um problema de decisão empresarial qualitativa. Revista de Administação de Empresas, Sāo Paulo v. 14, n. 4 , p. $79-84$, jul./ago. 1974.

10. A numerical algorithm for the resolution of Boolean equations. IEEE Transactions on Computers, y. 23 , n. 9 , p. 983-6, Sept. 1974 .

11. Métodos numéricos para a resolução de problemas lógicos. São Paulo: Blücher, 1993.

12. FAURE, E.; PAPIN, M.D.; KAULMANN, A. Cours de calcus booiéien appliqué. Paris: Albin Michel, 1970.

13. GRANA N. Lógica paraconsistente; una introduzione. Napoli, Loffredo, 1983.

14. HEGENBERG, L. Lógica: o cálculo sentencial. São Paulo Herder, EDUSP, 1972.

15. L. Lógica: o cálculo de predicados. São Pau10: Herder, EDUSP, 1973.
16. Lógica: exercicios $\|$ - dedução no cálculo sentencial. São Paulo: Pedagógica, 1976.

17. HILL, F.J: PETERSON, G.R. Introduction to switching theory and logical design. New York: John Willey, 1968

18. HUGHES, G. E., CRESSWELL, M.J. An introduction to modal logic. London: Methuen, 1972.

19. KLEENE. S.C. Mathematical logic. New York: John Wiley, 1967.

20. LAPPONI, J.C. Aplicaçāo da transtormada numérica na resolução de equaçōes booleanas. Electron, v. 7, ก. 40, p. 206-12, jul./ago, 1970

21.LEDLEY, R.S. Digital computer and control engineering. New York: McGraw Hill, 1960

22. MARANCA, A. P. G. L. Controladores com auto-sintonia difusa para sistemas de engenharia química. Săo Paulo: Escola Politécnica/Universidade de São Pau$10,199+110$ p. Dissertaçāo (Mestrado)

23. MARTINS, W. W. Contribuições ao estudo dos circuitos interruptores. São Paulo: Escola Politécnica/Universidade de São Paulo, 1 parte, 1964. 158p. Tese (Livre Docência).

$$
24 .
$$
Contribuiçôes ao estudo dos circuitos interruptores. Sã̃o Paulo: Escala Politécnica/Universidade de São Paula, 1967. $2^{2}$ parte, 194p. Tese (Cátedra).
25. Lógica Linguística. Ciência e Cultura. São Paulo, (I parte; v. 36, ค. 8, p. 1331-49, 1984; II parte: v. 37, n. 4 . p. $584-600,1985$; III parte: $v$. 39), n. 5/6, . 489-98, 1987.

26. MC CLUSKEY, E. J. Logic design principles. Englewood Cliffs: Prentice Hall, 1986

27. MENDELSON. E. Introduction to mathematical logic. New York: van Nostrand, 1964.

28. PESSOTA, R.C. C.L.P "Não-Von" a tompo real, matematicamente programável. São Paulo: Escola Politénica/Universidade de Sầo Paulo, 1993. 384p. Tese (Doutoramento)

29. ROTONDARO, I. G. A aplicaça da matemática booleana na obtenção dos sistemas de funçōes componentes de um sistema de funçōes São Paulo: Universidade Mackenzie, 1987. 155p. (Tese de Doutorado)

30. SKALA, H.J. On many valued logics, fuzzy sets, fuzzy logics and their applications. Fuzzy Sets and Systems, v.1, n. 2, p. 129-49, Abr. 1978

31. SMETS, P.; MANDANI, E. $H_{\text {.; }}$ DUBOIS, D.; PRADE, $H$. NOnstandard logic for automated reasoning. San Diego: Academic Press, 1988

32. TARSKI, A. Introduction to logic and to the methodology of deductive sciences. New York: Oxford University Press, 1965.

33. WHITESITT, J. E. Boolean algebra and its apolications. New York: Addison-Wesley, 1962. 